\title{
Internal Electric Fields Near Isolated Defects in Ionic Crystals
}

\author{
Herbert S. Bennett \\ Institute for Materials Research, National Bureau of Standards, Washington, D.C. 20234
}

(April 23, 1973)

\begin{abstract}
Integral representations for three distributions of internal electric fields near isolated defects in ionic crystals are given. These three distributions are the Gaussian distribution for electric fields associated with phonons, and two Holtsmark type distributions for electric fields associated with charged impurities when the Jahn-Teller effect is small and when it is large. Numerical values for the distributions and for the averages of squared-dipole matrix elements over them are tabulated in the conclusion.
\end{abstract}

Key words: Electric fields; $F$ centers; Gaussian function; Holtsmark function; isolated defects.

\section{Introduction}

The author and A. M. Stoneham [1] ${ }^{1}$ examined recently the effects of internal electric fields produced by lattice vibrations and charged impurities on the lifetimes of low-lying relaxed states of isolated defects. We computed in [1] by statistical methods the distributions of internal electric fields produced by phonons and charged impurities. These distributions are the Gaussian distribution for the phonons, a Holtsmark distribution for charged impurities when the JahnTeller effect is small, and a distribution related to the Holtsmark distribution when the Jahn-Teller effect is large. We then averaged the square of dipole-radiation matrix elements over these three distributions of internal electric fields. We did not present in [1] the numerical methods by which we evaluated the distributions of fields and the averages of squared-dipole matrix elements over the distributions to avoid distracting the reader from the major physical concepts.

Some researchers have expressed an interest in the derivations of the expansions appearing in the appendix to [1] and in the numerical methods used to evaluate the averaged matrix elements. The author now takes this opportunity to present the derivations and numerical methods used in [1]. Such mathematical procedures have applications to future studies on the temperature dependence of internal electric fields near defects.

Section 2 contains integral representations for the distributions of internal electric fields and series expansions of the distributions which are valid for large and small values of the fields. Evaluations of the square of dipole-radiation matrix elements averaged over these distributions appear in section 3. Finally, section 4 contains tables which give some representative numer- ical values for the distributions and the averages of squared-dipole matrix elements.

\section{Distributions of Fields}

The authors of [1] describe three classes of internal electric fields. The first class arises from the lattice vibrations. The second and third classes arise from the static fields associated with a random distribution of charged impurities in the host lattice. Some examples of such charged impurities are substitutional aliovalent ions, vacancies, and interstitials.

\subsection{Lattice Vibrations}

Bennett and Stoneham [1] compute the probability that the internal electric field associated with optical phonons has a value between $E$ and $E+d E$. They find that the probability for a harmonic lattice is proportional to the Gaussian distribution

$$
G(\gamma)=\pi^{-1 / 2} \exp \left(-\gamma^{2} / 4\right),
$$

where $\gamma=\left(2 E_{2}\right)^{-1 / 2} E$ is dimensionless and where $G(\gamma)$ is normalized to unity, $\int_{0}^{\infty} G(\gamma) d \gamma=1$. The quantity $E_{2}$ is the square of a characteristic electric field for the lattice. The Gaussian function $G(\gamma)$ also has the following integral representations;

$$
G(\gamma)=\frac{2}{\pi \gamma} \int_{0}^{\infty} \exp \left(-x^{2} / \gamma^{2}\right) \cos x d x
$$

and

$$
G(\gamma)=\frac{2}{\pi} \int_{0}^{\infty} \exp \left(-y^{2}\right) \cos (\gamma y) d y
$$


of squared-dipole radiation matrix elements. Because eq (1) is in a convenient form, it is not necessary to derive expansions which are valid for large $\gamma$ and for small $\gamma$. This is not the case for the remaining two distributions, however.

\subsection{Static Fields}

A charged impurity can perturb lattice defects such as $F$ centers by a number of different mechanisms. One such mechanism is the electric field produced by the impurity. The authors of [1] also compute the distribution in the magnitude of the electric field $|\mathbf{E}|$ and the distribution in the projection of $\mathbf{E}$ onto a specific direction.

When the Jahn-Teller effect is small, they find that the distribution in the magnitude of $|\mathbf{E}|=E$ is proportional to the Holtsmark function $H(\beta)$,

$$
H(\beta)=\frac{2}{\pi \beta} \int_{0}^{\infty} d x x \sin x \exp \left\{-(x / \beta)^{3 / 2}\right\},
$$

where $\beta=a^{-2 / 3} E$ is dimensionless, and where $H(\beta)$ is normalized to unity, $\int_{0}^{\infty} H(\beta) d \beta=1$. The quantity $a^{2 / 3}$ is a characteristic electric field associated with the charged impurities imbedded in a dielectric host. Representation (4) is the convenient one from which to generate an expansion valid for large $\beta$. The representation,

$$
H(\beta)=\frac{2 \beta}{\pi} \int_{0}^{\infty} d y y \sin (\beta y) \exp \left(-y^{3 / 2}\right),
$$

is the convenient representation from which to generate an expansion valid for small $\beta$ and from which to evaluate averages of the dipole-radiation matrix element.

When the Jahn-Teller effect is large, the distribution in magnitude of the projection of $\mathbf{E}$ onto a specific direction is proportional to a distribution $H^{\prime}(\beta)$ related to the Holtsmark distribution; namely,

$H^{\prime}(\beta)=\frac{1}{\pi \beta} \int_{0}^{\infty} d x \cos x \exp \left\{-(x / \beta)^{3 / 2}\right\}$,

where $H^{\prime}(\beta)$ has the normalization, $2 \int_{0}^{\infty} H^{\prime}(\beta) d \beta=1$.

Both functions $H$ and $H^{\prime}$ are called Holtsmark functions. The function $H^{\prime}$ is intermediate between a Lorentzian and a Gaussian distribution. Representation (6) is convenient for developing an expansion valid for large $\beta$ and the following representation,

$$
H^{\prime}(\beta)=\frac{1}{\pi} \int_{0}^{\infty} d y \cos (\beta y) \exp \left(-y^{3 / 2}\right),
$$

readily leads to an expansion valid for small $\beta$. It is also the useful one from which to evaluate averages of squared-dipole radiation matrix elements.

When $\beta$ has intermediate values near unity, the author finds that it is necessary to evaluate numerically the integrals in eqs (4) to (7). It is convenient for him to integrate by Gaussian quadratures with Legendre polynomials. The Legendre polynomials require a finite interval of integration. The semi-infinite interval $0 \leqslant x \leqslant \infty$ is approximated by the interval $10^{-5} \beta \leqslant x \leqslant$ 6.34 $\beta$ when eqs (2), (4), and (6) are evaluated numerically in the present work. Expansions valid for $\beta \approx 1$ coverage slowly and are not competitive with the above numerical integration procedures when computation time is important. Likewise, asymptotic expansions which converge rapidly when $\beta$ is very large or very small are more efficient than the above numerical integration procedures for such extreme values of $\beta$. The next two subsections contain summaries of the derivation for such series expansions.

\subsection{Expansions for Large $\beta$}

In this subsection, expansions for the distributions $H(\beta)$ and $H^{\prime}(\beta)$ which are valid for $\beta>>1$ are derived. The representation (4) for $H(\beta)$ and the representation $\left(\epsilon_{)}\right)$for $H^{\prime}(\beta)$ are convenient ones from which to begin. When $\alpha=\beta^{-3 / 2}$, the distributions become

$$
H(\beta)=(2 / \pi \beta) I(\alpha),
$$

and

$$
H^{\prime}(\beta)=(1 / \pi \beta) J(\alpha)
$$

where

$$
I(\alpha)=\int_{0}^{\infty} d x x \sin x \exp \left(-\alpha x^{3 / 2}\right)
$$

and

$$
J(\alpha)=\int_{0}^{\infty} d x \cos x \exp \left(-\alpha x^{3 / 2}\right) .
$$

Because the quantity $\alpha$ approaches zero in the limit as $\beta$ approaches infinity, Taylor series expansions about $\alpha=0$ become useful representations for the functions $I(\alpha)$ and $J(\alpha)$.

Using the following limits

$$
x \sin x=\frac{1}{2 i}\left(-\operatorname{Lim}_{p \rightarrow-i^{+}}+\underset{p \rightarrow i^{+}}{\operatorname{Lim}}\right)\left(\frac{\partial}{\partial p} e^{-p x}\right),
$$

and

$$
\cos x=\frac{1}{2}\left(\operatorname{Lim}_{p \rightarrow-i^{+}}+\underset{p \rightarrow i^{+}}{\operatorname{Lim}}\right) e^{-p x},
$$


and the Laplace transformed function

$$
f(p ; \alpha)=\int_{0}^{\infty} d x \exp \left(-p x-\alpha x^{3 / 2}\right),
$$

we write eqs (10) and (11) in the forms

$$
I(\alpha)=\frac{1}{2 i}\left(-\operatorname{Lim}_{p \rightarrow-i^{+}}+\underset{p \rightarrow i^{+}}{\operatorname{Lim}}\right) \frac{\partial}{\partial p} f(p ; \alpha),
$$

and

$$
J(\alpha)=\frac{1}{2}\left(\underset{p \rightarrow-i^{+}}{\operatorname{Lim}}+\underset{p \rightarrow i^{+}}{\operatorname{Lim}}\right) f(p ; \alpha) .
$$

Expanding $f(p ; \alpha)$ in a Taylor's series about $\alpha=0$ and noting the Laplace transform

$$
\int_{0}^{\infty} d x \exp (-p x) x^{s}=\frac{\Gamma(s+1)}{p^{s+1}},
$$

where $\Gamma(s+1)$ is the gamma function,

$$
\Gamma(s+1)=\int_{0}^{\infty} d z z^{s} \exp (-z),
$$

and where $s+1>0$, we have

$I(\alpha)=\frac{1}{2 i}\left(-\operatorname{Lim}_{p \rightarrow-i^{+}}+\underset{p \rightarrow i^{+}}{\operatorname{Lim}}\right) \sum_{n=0}^{\infty}$

$$
\frac{(-\alpha)^{n}}{n !}\left(\frac{3 n}{2}+1\right) \frac{\Gamma\left(\frac{3 n}{2}+1\right)}{p^{(3 n / 2)}}
$$

and

$J(\alpha)=\frac{1}{2}\left(\operatorname{Lim}_{p \rightarrow-i^{+}}+\operatorname{Lim}_{p \rightarrow i^{+}}\right) \sum_{n=0}^{\infty} \frac{(-\alpha)^{n}}{n !} \frac{\Gamma\left(\frac{3 n}{2}+1\right)}{p^{(3 n / 2)+1}}$.

Inserting $-i=\exp (-\pi i / 2)$ and $i=\exp (\pi / 2)$ into the above equations gives us

$I(\alpha)=-\sum_{n=0}^{\infty} \frac{(-\alpha)^{n}}{n !} \Gamma\left(\frac{3 n}{2}+1\right)\left(\frac{3 n}{2}+1\right) \sin \left(\frac{3 \pi n}{4}\right)$,

and

$J(\alpha)=\sum_{n=0}^{\infty} \frac{(-\alpha)^{n}}{n !} \Gamma\left(\frac{3 n}{2}+1\right) \cos \left\{\left(\frac{3 n}{2}+1\right) \frac{\pi}{2}\right\}$.

The expansions for $H(\beta)$ and $H^{\prime}(\beta)$ which are valid when $\beta \gg 1$, then become

$H(\beta)=\frac{2}{\pi \beta}\left\{\left(\frac{\pi}{2}\right)^{1 / 2} \sum_{m=0}^{\infty} \frac{(6 m+5)}{2} \frac{(6 m+3)}{2}\right.$

$$
\begin{array}{r}
\frac{(6 m+1)}{2} \ldots \frac{1}{2} \frac{\alpha^{2 m+1}}{(2 m+1) !}+\sum_{m=0}^{\infty} \frac{(6 m+8)}{2} \frac{(6 m+6)}{2} \\
\left.\quad \frac{(6 m+4)}{2} \ldots \frac{2}{2} \frac{\alpha^{2 m+2}}{(2 m+2) !}\right\},
\end{array}
$$

or

$$
\begin{array}{r}
H(\beta) \underset{\beta \rightarrow \infty}{\rightarrow} \frac{15}{8}\left(\frac{2}{\pi}\right)^{1 / 2} \beta^{-5 / 2}+\frac{24}{\pi} \beta^{-4}+\frac{10395}{384} \\
\left(\frac{2}{\pi}\right)^{1 / 2} \beta^{-11 / 2}+\ldots ;
\end{array}
$$

and

$$
\begin{array}{r}
H^{\prime}(\beta)=\frac{1}{\pi \beta}\left\{\left(\frac{\pi}{2}\right)^{1 / 2} \sum_{m=0}^{\infty} \frac{(6 m+3)}{2} \frac{(6 m+1)}{2} \ldots\right. \\
\frac{1}{2} \frac{\alpha^{2 m+1}}{(2 m+1) !}+\sum_{m=0}^{\infty} \frac{(6 m+6)}{2} \frac{(6 m+4)}{2} \ldots \\
\left.\frac{2}{2} \frac{\alpha^{2 m+2}}{(2 m+2) !}\right\},
\end{array}
$$

or

$$
H^{\prime}(\beta) \underset{\beta \rightarrow \infty}{\rightarrow}\left\{\frac{3}{4(2 \pi)^{1 / 2}} \beta^{-5 / 2}+\frac{3 \beta^{-4}}{\pi}+\frac{945 \beta^{-11 / 2}}{192(2 \pi)^{1 / 2}}+\ldots\right\}
$$

\subsection{Expansions for Small $\beta$}

In this subsection, expansions for the distributions $H(\beta)$ and $H^{\prime}(\beta)$ which are valid for $\beta \ll 1$ are derived. Substituting $y=x^{3 / 2}$ in eq (12) and representing exp $\left(-p y^{2 / 3}\right)$ by the Taylor's series expansion

$$
\exp \left(-p y^{2 / 3}\right)=\sum_{n=0}^{\infty} \frac{\left(-p y^{2 / 3}\right)^{n}}{n !},
$$

we obtain

$$
f(p ; \alpha)=\frac{2}{3} \sum_{n=0}^{\infty} \frac{(-p)^{n}}{n !} \frac{\Gamma\left(\frac{2 n}{3}+\frac{2}{3}\right)}{\alpha^{[2(n+1)] / 3}} .
$$

Applying the operations which are indicated in eqs (13) and (14) to the representation (22) for $f(p ; \alpha)$ gives us

$$
I(\alpha)=\frac{2}{3} \sum_{n=2}^{\infty} \frac{\sin \left(\frac{\pi(n-1)}{2}\right)}{(n-1) !} \frac{\Gamma\left(\frac{2 n}{3}+\frac{2}{3}\right)}{\alpha^{[2(n+1)] / 3}},
$$

and

$$
J(\alpha)=\frac{2}{3} \sum_{n=0}^{\infty} \frac{\cos (\pi n / 2)}{n !} \frac{\Gamma\left(\frac{2 n}{3}+\frac{2}{3}\right)}{\alpha^{[2(n+1)] / 3}} .
$$


Hence, the expansions for small $\beta$ are

$$
H(\beta)=\frac{4}{3 \pi} \sum_{m=0}^{\infty}(-1)^{m} \frac{\beta^{2 m+2}}{(2 m+1) !} \Gamma\left(\frac{4 m+6}{3}\right),
$$

or

$H(\beta) \underset{\beta \rightarrow 0}{\rightarrow} \frac{4}{3 \pi}\left[\beta^{2}-\frac{\beta^{4}}{6} \Gamma\left(\frac{10}{3}\right)+\frac{\beta^{6}}{120} \Gamma\left(\frac{14}{3}\right)+\ldots\right]$

and

$H^{\prime}(\beta)=\frac{2}{3 \pi} \sum_{m=0}^{\infty}(-1)^{m} \frac{\beta^{2 m}}{(2 m) !} \Gamma\left(\frac{4 m+2}{3}\right)$,

or

$H^{\prime}(\beta) \underset{\beta \rightarrow 0}{\rightarrow} \frac{2}{3 \pi}\left(\Gamma\left(\frac{2}{3}\right)-\frac{\beta^{2}}{2}+\frac{\beta^{4}}{24} \Gamma\left(\frac{10}{3}\right)+\ldots\right)$.

The first two terms of eqs (18) and (26) for the distribution $H(\beta)$ appear also in [3]. To the author's knowledge, the expansions given above for the distribution $H^{\prime}(\beta)$ have not been reported previously.

\section{Averages of Squared-Dipole Matrix Elements}

Averaging the square of the dipole-radiation matrix elements over the distributions $G(\gamma), H(\beta)$, and $H^{\prime}(\beta)$ provides us with estimates of the effects which electric fields have on the lifetimes of relaxed $F$-center states. Discussions of these effects appear in [1]. It has been shown therein that the averages of the squares of the dipole radiation matrix elements over the distributions $G, H$, and $H^{\prime}$ are proportional to the following dimensionless expressions:

$$
M_{ \pm}^{2}[G ; \gamma]=\frac{1}{2}\left[1 \pm \int_{0}^{\infty}\left\{1+(\alpha / \gamma)^{2}\right\}^{-1 / 2} G(\alpha) d \alpha\right]
$$

$M_{ \pm}^{2}[H ; \beta]=\frac{1}{2}\left[1 \pm \int_{0}^{\infty}\left\{1+(\alpha / \beta)^{2}\right\}^{-1 / 2} H(\alpha) d \alpha\right]$,

and

$M_{ \pm}^{2}\left[H^{\prime} ; \beta\right]=\frac{1}{2}\left[1 \pm 2 \int_{0}^{\infty}\left\{1+(\alpha / \beta)^{2}\right\}^{-1 / 2} H^{\prime}(\alpha) d \alpha\right]$.

The ordering of the energy levels of the $F$ center determines whether the plus or minus sign is used. The details are discussed in [1].

The integrals appearing in eqs (29) to (31) are evaluated by two methods. Both methods employ numerical integration by Gaussian quadratures [2]. The first method is more straightforward than the second method; but it requires in some cases more time for computation than the second method requires. In the first method, the distributions $G(\alpha), H(\alpha)$, and $H^{\prime}(\alpha)$ are computed explicitly. Values of $G(\alpha)$ for the region $0 \leqslant \alpha \leqslant \infty$ are given directly by eq (1). Values of $H(\alpha)$ and $H^{\prime}(\alpha)$ are given by the following prescription. They are obtained when $1 \leqslant \alpha \leqslant 6$ by applications of Gaussian quadratures to the respective representations (4) and (6). The respective expansions (18) and (20) for $H(\alpha)$ and $H^{\prime}(\alpha)$ are used when $\alpha \gg 6$; and the respective expansions (26) and (28) are used when $\alpha \ll 1$. These values for the distributions $G(\alpha), H(\alpha)$, and $H^{\prime}(\alpha)$ then are inserted. into the integrands of eqs (29) to (31). Finally, the integrals are evaluated by Gaussian quadratures.

The second method does not involve explicit evaluations of the distributions. Instead, the representations (3), (5), and (7) are inserted respectively into eqs (29) to (31), namely,

$$
\begin{gathered}
M_{ \pm}^{2}[G ; \gamma]=\frac{1}{2} \pm \frac{1}{\pi} \int_{0}^{\infty} d \alpha \int_{0}^{\infty} d y\left\{1+(\alpha / \gamma)^{2}\right\}^{-1 / 2} \\
\exp \left(-y^{2}\right) \cos (\alpha y),
\end{gathered}
$$

$$
\begin{array}{r}
M_{ \pm}^{2}[H ; \beta]=\frac{1}{2} \pm \frac{1}{\pi} \int_{0}^{\infty} d \alpha \int_{0}^{\infty} d y\left\{1+(\alpha / \beta)^{2}\right\}^{-1 / 2} \\
\quad \exp \left(-y^{3 / 2}\right) \alpha y \sin (\alpha y),
\end{array}
$$

and

$$
\begin{gathered}
M_{ \pm}^{2}\left[H^{\prime} ; \beta\right]=\frac{1}{2} \pm \frac{1}{\pi} \int_{0}^{\infty} d \alpha \int_{0}^{\infty} d y\left\{1+(\alpha / \beta)^{2}\right\}^{-1 / 2} \\
\quad \exp \left(-y^{3 / 2}\right) \cos (\alpha y) .
\end{gathered}
$$

The integrations over $\alpha$ then are performed by referring to several equations in section 9.6 of [2]. The results are

$$
\int_{0}^{\infty}\left\{1+(\alpha / \beta)^{2}\right\}^{-1 / 2} \cos (\alpha y) d \alpha=\beta K_{0}(\beta y),
$$

and

$\int_{0}^{\infty}\left\{1+(\alpha / \beta)^{2}\right\}^{-1 / 2} \alpha \sin (\alpha y) d \alpha=\beta^{2} K_{1}(\beta y)$,

where $\beta y \geqslant 0$ and where $K_{0}$ and $K_{1}$ denote, respectively, the modified Bessel functions of zero and first order. Insertion of eqs (35) and (36) into eqs (32) to (34) yields the following expressions:

$$
\begin{aligned}
& M_{ \pm}^{2}[G ; \gamma]=\frac{1}{2} \pm \frac{\gamma}{\pi} \int_{0}^{\infty} d y \exp \left(-y^{2}\right) K_{0}(\gamma y), \\
& M_{ \pm}^{2}[H ; \beta]=\frac{1}{2} \pm \frac{\beta^{2}}{\pi} \int_{0}^{\infty} d y \exp \left(-y^{3 / 2}\right) y K_{1}(\beta y),
\end{aligned}
$$

and 
$M_{ \pm}^{2}\left[H^{\prime} ; \beta\right]=\frac{1}{2} \pm \frac{\beta}{\pi} \int_{0}^{\infty} d y \exp \left(-y^{3 / 2}\right) K_{0}(\beta y)$.

The modified Bessel functions are evaluated according to their polynomial approximations given in section 9.8 of [2]. The final step of the second method consists of the numerical integration of eqs (37) to (39) by Gaussian quadratures.

Eash of these two methods has its advantages. The decision as to which method is most suitable depends upon the values of $\gamma$ and $\beta$ and upon the other types of calculations included in the computer program.

\section{Conclusions}

We conclude this paper by presenting some numerical results. Table 1 gives values of $G(\beta), H(\beta)$, and $H^{\prime}(\beta)$ for $0 \leqslant \beta \leqslant 10.0$. Table 2 contains the values of $M_{+}^{2}$ for each of the three distribution functions. The respective values of $M_{-}^{2}$ follow from the relation $M_{-}^{2}=1-M_{+}^{2}$.

TABLE 1. The distribution functions $\mathrm{G}(\beta), \mathrm{H}(\beta)$ and $\mathrm{H}^{\prime}(\beta)$

\begin{tabular}{c|c|c|c}
\multicolumn{4}{|c}{ All quantities are dimensionless. } \\
\hline \hline$\beta$ & $G(\beta)$ & $H(\beta)$ & $H^{\prime}(\beta)$ \\
\hline 0.0 & 0.56 & 0.00 & 0.29 \\
.04 & .56 & .00 & .29 \\
.1 & .56 & .004 & .29 \\
.4 & .54 & .06 & .27 \\
1.0 & .44 & .27 & .20 \\
4.0 & .01 & .08 & .01 \\
10.0 & .00 & .01 & .00 \\
\hline
\end{tabular}

TABLE 2. The square of the dipole-matrix elements averaged over the three distributions $\mathrm{G}, \mathrm{H}$, and $\mathrm{H}^{\prime}$

\begin{tabular}{c|c|c|c}
\multicolumn{5}{c}{ All quantities are dimensionless. } \\
\hline \hline$\beta$ & $M_{+}^{2}[G ; \gamma=\beta]$ & $M_{+}^{2}[H ; \beta]$ & $M_{+}^{2}\left[H^{\prime} ; \beta\right]$ \\
\hline 0.01 & 0.51 & 0.50 & 0.51 \\
.04 & .54 & .51 & .54 \\
.1 & .59 & .53 & .59 \\
.4 & .74 & .61 & .73 \\
1.0 & .85 & .72 & .84 \\
4.0 & .97 & .91 & .96 \\
10.0 & .99 & .97 & .99 \\
\hline
\end{tabular}

The author thanks A. M. Stoneham and L. V. Spencer for helpful discussions. He wishes to thank the personnel of the Aspen Center for Physics, where portions of the manuscript were written, for their kind hospitality.

\section{References}

[1] Bennett, H. S., and Stoneham, A. M., Phys. Rev. B 6, 3086 (1972).

[2] Handbook of Mathematical Functions, edited by M. Abramowitz and I. A. Stegun, Nat. Bur. Stand. (U.S.), Appl. Math. Ser. 55, (1965), p. 887 and p. 916.

[3] Chandrasekhar, S., and Van Neumann, J., Astrophysical Journal 95, 489 (1942). These authors state that the coefficients in the series expansion for $H(\beta)$ have been tablulated by S. Ver Weij, Pub. Ap. Inst. Amsterdam, No. 5, Table 3 (1936). The latter author gives the first 20 terms of the function $H(\beta)$ for $\beta$ very large and the first 36 terms of the function $H(\beta)$ for $\beta$ very small. 